\title{
Magnificación en la terapia endodóncica mediante el microscopio operatorio.
}

\section{Magnification in endodontic therapy through the operatory microscope.}

\author{
Delia Nallely Jiménez-Delgadillo,* Jairo Mariel-Cárdenas, \\ Francisco Javier Gutiérrez-Cantú, ${ }^{\ddagger}, \|$ Ricardo Oliva-Rodríguez ${ }^{\ddagger}, * *$
}

\section{RESUMEN}

La microendodoncia involucra la visualización a través de un microscopio operatorio de todas las fases del tratamiento de conductos y procedimientos de cirugía apical y correctiva por parte del endodoncista. Existe sobrada evidencia acerca de las mejoras que puede aportar la magnificación al tratamiento; la literatura demuestra que la capacidad del operador mejora si su visión del campo gana claridad y precisión, ambos recursos pueden ser proporcionados por el microscopio operatorio, aunado a que posibilita diagnósticos más certeros junto con mejoras en el pronóstico, lo que permite evitar posibles complicaciones. La calidad de los tratamientos endodóncicos involucra infinidad de factores, cada uno relevante en sí mismo pero, en determinados casos, el microscopio puede significar la diferencia entre un tratamiento exitoso o un fracaso clínico. En la actualidad, se ha convertido en un tema de lo más relevante, por lo que el objetivo del presente trabajo es revisar la literatura con el fin de ayudar al entendimiento basado en evidencia científica de los criterios que determinan la relevancia del uso del microscopio en el ámbito endodóncico.

Palabras clave: Microendodoncia, microscopio operatorio, magnificación.

\section{ABSTRACT}

Microendodontics involves the visualization through an operating microscope of all phases of root canal treatment and apical and corrective surgery procedures by the endodontist. There is plenty of evidence about the improvements that magnification can provide, the literature shows that the operator's ability improves if his vision of the field gains clarity and precision, both resources can be provided by the operating microscope, added to the fact that it enables more accurate diagnoses together with improvements in the prognosis allowing to avoid possible complications. The quality of endodontic treatments involves countless factors, each relevant in itself, but in certain cases the microscope can mean the difference between a successful treatment or a clinical failure. At present, it has become a very relevant topic, so the objective of this work is to review the literature in order to help understand the criteria that determine the relevance of the use of the microscope in the endodontic field based on scientific evidence.

Keywords: Microendodontics, operating microscope, magnification.

\section{INTRODUCCIÓN}

$\mathrm{L}$ os inicios de la invención del microscopio datan del siglo XVI, con Zacharias Janssen, aunque es Leeuwenhoek quien creó microscopios rudimentarios con una resolución aceptable. ${ }^{1}$ El microscopio se empezó a utilizar en 1957 en otorrinolaringología. Carl Nylen creó un microscopio monocular para realizar cirugía de oído. ${ }^{2}$ Esto influyó en el avance de la cirugía, ya que a partir de ese suceso otras especialidades fueron incluyendo

\footnotetext{
* Cirujano Dentista, Universidad de Guadalajara, CUALTOS. Tepatitlán de Morelos, Jalisco.

‡ Facultad de Estomatología, Universidad Autónoma de San Luis Potosí. México.

$\S$ Doctor en Ciencias Biológicas, Profesor Investigador, Departamento de Morfología. Universidad Autónoma de San Luis Potosí. México.

^ Especialista en Ortodoncia, Profesor Investigador, Departamento de Morfología. Universidad Autónoma de San Luis Potosí. México.

" Doctor en Ciencias Biológicas, Profesor Investigador, Maestría en Ciencias Odontológicas. Universidad Autónoma de San Luis Potosí. México.

** Doctor en Ciencias Biológicas, Profesor Investigador, Maestría en Endodoncia. Universidad Autónoma de San Luis Potosí. México.

Recibido: 16 de agosto de 2020. Aceptado: 18 de mayo de 2021.
}

Citar como: Jiménez-Delgadillo DN, Mariel-Cárdenas J, Sánchez-Meraz W, Gutiérrez-Cantú FJ, Oliva-Rodríguez R. Magnificación en la terapia endodóncica mediante el microscopio operatorio. Rev ADM. 2021; 78 (3): 176-180. https://dx.doi.org/10.35366/100076

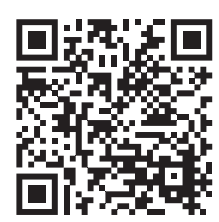


su uso y se comenzó a buscar mejorar las condiciones ópticas. Fue hasta 1978 cuando Apotheker y Jako adaptaron un microscopio para uso en odontología, aunque inicialmente los odontólogos no identificaban sus ventajas como herramienta clínica. ${ }^{3}$ Después, en 1992, Carr diseñó el primer microscopio operatorio dental (MOD), que definiría las características de los MOD actuales, los cuales incluyen sistemas de iluminación coaxial con luces halógenas de alta intensidad o de xenón, ópticas con rangos de magnificación adaptados a la cavidad oral que permiten trabajar a magnificaciones que oscilan entre $6 x y$ 20x; con binoculares inclinables empotrados en sistemas más livianos que permiten una movilidad óptima para ver cualquier diente o cuadrante de la boca. ${ }^{4,5}$ En 1998, la Asociación Dental Americana estableció una norma que promueve que los programas de especialización en endodoncia incluyan formación y aprendizaje del uso de microscopio operatorio, creando nuevos estándares para acreditación.

Funcionamiento. Existen tres conceptos clave que ayudan a dimensionar la utilidad del microscopio: la magnificación, la iluminación y la documentación. La magnificación es determinada por la potencia del ocular, la longitud focal de los binoculares, el regulador de los factores de aumento y la longitud focal del objetivo. La iluminación de los microscopios es coaxial con la línea de visión, lo que permite que se pueda ver el campo de visión sin sombras, al ser paralela la luz, se evita la convergencia ocular, manteniendo los ojos en reposo, y así poder realizar procedimientos prolongados sin fatiga ocular. Además, a través de diferentes aditamentos, ofrece la capacidad para la documentación de todo el procedimiento clínico. ${ }^{5}$

Conviene tener en cuenta para entender mejor el funcionamiento de un microscopio y así poder seleccionar el más acorde a un procedimiento estándar tres conceptos básicos, que son:

1. Longitud focal. La cual determina la distancia que debe haber entre la lente y el campo quirúrgico. La longitud focal objetiva varía de 100 a $400 \mathrm{~mm}$. Se recomienda de $200 \mathrm{~mm}$ para el uso endodóncico.

2. Campo de visión. El campo de visión es el área de operación que se ve a través de las lupas. El ancho de campo está relacionado con el diámetro de la lupa, el diseño óptico y el poder de aumento.

3. Profundidad de campo. Es la capacidad del sistema de lentes para enfocarse en objetos que están cerca o lejos sin tener que cambiar la posición de la lupa. Generalmente, las lentes están disponibles en aumentos de X6.3; X10; X12.5; X16; X20.54. ${ }^{6}$ La profundidad de campo es inversamente proporcional al aumento total. Si la ampliación total aumenta, la profundidad del campo disminuye.

\section{APLICACIONES}

El microscopio operatorio en endodoncia se ha convertido en un recurso fundamental, su empleo no cambia las técnicas endodóncicas del operador, aporta precisión a las mismas, y así facilita conseguir el objetivo de tratamientos exitosos y predecibles.

Shanelec y Tibbets indican que al trabajar sin aumento se pueden efectuar movimientos de 1 a $2 \mathrm{~mm}$ a la vez; y cuando se emplea un aumento de 20x, los movimientos pueden ser de 10 a $20 \mu \mathrm{m}$, lo que concluye que la exactitud del tratamiento depende básicamente de los ojos del operador más que de sus manos, lo que lleva a tomar en cuenta la extensión de la vista que puede significar el MOD.

Se recomiendan magnificaciones bajas, en el rango de las $4 x$ para actividades en cirugía como: orientación inicial e inspección del sitio quirúrgico, osteotomía inicial, alineación de las puntas ultrasónicas en la retro-preparación cavitaria y para la colocación de sutura y retiro de ésta. Los aumentos medios, cercanos a 10x son útiles en el tratamiento endodóncico en la fase de acceso, identificación de fracturas, retiro de instrumentos separados y en la fase de obturación; mientras que en cirugía apical son útiles para conseguir hemostasia en sitios puntuales, identificar conductos, remoción de tejido, resección del ápice, retropreparación y obturación del extremo apical, así como en la inspección a detalle de la superficie radicular; mientras que los aumentos altos en el orden de las 20x muestran su utilidad en la ubicación e identificación de orificios de entrada de conductos calcificados, en la búsqueda de fisuras e identificación de detalles anatómicos finos; en cirugía, permiten inspeccionar la superficie radicular, la retro-preparación y obturación cavitaria, así como para identificar detalles anatómicos finos que de otro modo pueden ser pasados por alto. La documentación de casos tanto quirúrgicos como no quirúrgicos se procura hacer en magnificaciones altas. ${ }^{7}$

\section{DIAGNÓSTICO}

La mayoría de los endodoncistas estarían de acuerdo en que el diagnóstico es uno de los aspectos más desafiantes en endodoncia. Un especialista debe ser un experto en diagnóstico, por lo que cualquier equipo que ayude a tal 
fin es útil, y el microscopio definitivamente cumple con este criterio. ${ }^{6}$ El microscopio, al igual que los instrumentos, radiografías, aerosoles para pruebas de vitalidad y tintas son un recurso auxiliar que puede llegar a generar una diferencia entre un diagnóstico u otro. Genera imágenes que ningún ojo clínico podría distinguir. La visión del odontólogo puede ser capaz de distinguir detalles finos, pero cuando una imagen se magnifica, aparece evidencia microscópica de signos patológicos importantes que son invisibles o no comprendidos a menos de $12 x{ }^{8}$ El aumento del campo de trabajo y la iluminación que da el MOD durante el diagnóstico es importante, sobre todo para reconocer detalles de la anatomía dental y de restauraciones, obturaciones con filtración, así como posibles fisuras o fracturas. ${ }^{2}$

Aperturas mínimamente invasivas. Los objetivos de la preparación de la cavidad de acceso buscan obtener una entrada en línea recta, conservar la mayor estructura dental sana y quitar el techo de la cámara pulpar para exponer y eliminar los cuernos pulpares. ${ }^{8}$ El acceso sin obstrucciones al sistema de conductos radicular y la identificación de estructuras intrincadas de su anatomía son clave para una terapia exitosa y el microscopio genera un campo de trabajo preciso. Un acceso adecuado permite visualizar en línea recta cada orificio de entrada de los conductos radiculares, lo que permite que la llegada de las soluciones irrigantes sea directa, la instrumentación más cómoda, la desinfección real y que disminuya enormemente la posibilidad de perforaciones en el piso cameral. ${ }^{8}$ Manejar este tipo de situaciones sin magnificación o una buena iluminación representa un reto. Se puede lograr, pero el número de errores aumenta.

Localización de conductos radiculares. La localización de conductos representa una importante indicación para el uso del microscopio, este punto puede generar una de las aportaciones más destacadas, pues la localización de conductos accesorios crea un pronóstico favorable en tratamientos endodóncicos a largo plazo, evitando reinfecciones.

El éxito del tratamiento endodóncico está relacionado directamente con el conocimiento de la anatomía interna del diente por parte del endodoncista. ${ }^{9}$ A pesar de tener en cuenta que los molares generan retos importantes, cada órgano dental representa problemáticas distintas, se sabe que el complejo pulpar en cada órgano es diferente y existen referencias básicas anatómicas que deben respetarse siempre. Mageste Duque evaluó incisivos inferiores permanentes ya tratados endodóncicamente en busca de conductos no tratados, comparando el método visual-radiográfico con la visualización con y sin MOD, esto mostró que la técnica radiográfica permitió su localización en $70.59 \%$ de los dientes, la evaluación visual sin MOD únicamente $\mathbf{1 1 . 7 6 \% ~ y ~ l a ~ e v a l u a c i o ́ n ~ c o n ~ M O D ~ e n ~}$ $47.05 \%$, lo cual concluye que la asociación de técnicas radiográficas y la magnificación visual obtenida con MOD se muestran como los aliados clínicos más efectivos para la localización de conductos. ${ }^{9}$ Monteiro Bramante demostró que la radiografía distorradial y el empleo del microscopio clínico ofrecen una mayor posibilidad de visualización del conducto mesiopalatino, aumentando hasta en $10 \%$ su detección. ${ }^{10}$ De la misma manera, Sánchez G en un estudio in vitro de localización de conductos radiculares donde comparó visión directa con visualización con microscopio operatorio, coincide con los resultados mencionados anteriormente, con un aumento de $11 \%$ para la localización de conductos accesorios. ${ }^{8}$

Recuperación de instrumentos fracturados en conductos. Como se ha mencionado, la magnificación y la iluminación se vuelven fundamentales en casos complicados, en accidentes o en el manejo de iatrogenias. Jadhav en un caso clínico de un canino mandibular de dos raíces y tres conductos con un instrumento fracturado, destacó la utilidad del microscopio para observarlo. ${ }^{11}$ Gencoglu menciona que la eliminación de los fragmentos requiere mucho tiempo, es arriesgada y tiene un éxito limitado; tras lo anterior, determinó que los ultrasonidos con la ayuda de un microscopio dental operatorio tienen más éxito en la extracción de instrumentos fracturados que los métodos convencionales. ${ }^{12}$

Sellado de perforaciones. El MOD es un instrumento de indudable valor para la reparación de perforaciones iatrogénicas donde el pronóstico depende de la calidad del sellado. De acuerdo con Espinosa, en el seguimiento de dos casos con perforaciones a cinco años, mostró resultados satisfactorios, ya que debido a la magnificación visual del campo pudo observar la perforación en ambos casos e, incluso, apreciar la presencia de tejido de granulación. ${ }^{13}$ Es de gran relevancia la visibilidad adecuada proporcionada por los microscopios clínicos para llevar a cabo esta clase de procedimientos.

Microcirugía. Algunas de las ventajas que aporta el microscopio operatorio a los procedimientos quirúrgicos son: osteotomías más pequeñas, biseles de menor angulación, conservación de más hueso cortical y estructura radicular, la inspección de la superficie radicular con iluminación y gran aumento que identifica detalles anatómicos y posibles causas del fracaso endodóncico. La poca visibilidad y el tamaño reducido del campo operatorio son factores que pueden influir en el pronóstico de estos tratamientos, donde la eliminación total de la 
lesión periapical es particularmente importante para mejorarlo. ${ }^{14}$ Con el surgimiento del microscopio operatorio y una gama de tecnologías asociadas, algunos autores plantean que el pronóstico a largo plazo de este tipo de tratamiento ha mejorado ampliamente, en comparación con la técnica convencional; aunque otros como Giménez del Arco mencionan que aunque un microscopio puede ser de gran utilidad, los resultados exitosos a largo plazo son consecuencia de una correcta selección del caso, estudio previo, conocimiento y aplicación de las técnicas quirúrgicas bajo protocolos adecuados en conjunto con tomografías computarizadas, puntas ultrasónicas, microespejos y materiales de retro-obturación adecuados. ${ }^{15}$ Espinosa Torres, presenta un accidente en furcación resuelto con microcirugía en un caso con control a 30 meses, en el que con una magnificación de 10x, y gracias a la claridad de imagen proporcionada por el microscopio, se pueden identificar las estructuras radiculares y el trabeculado óseo, y no comprometer la estructura dental al realizar el corte; así concluyó que la microcirugía permite evitar comprometer estructuras adyacentes a las zonas afectadas. ${ }^{16}$ Taschieri comparó el uso de lupas con el microscopio en cirugía endodóncica con seguimiento a uno y cuatro años, lo que mostró una tasa de éxito de $91.7 \%$ a un año y de $90.5 \%$ a cuatro años para el grupo que usó lupas; mientras que para el que usó microscopio fue de $91.4 \%$ a un año y de $93.3 \%$ a los cuatro años. Esto indica que el uso del microscopio puede ser más favorable aunque no de manera significativa, resaltando que el éxito en estos tratamientos con magnificación sobrepasó el $90 \% .{ }^{17}$ Es importante mencionar que si el acceso es limitado para la cirugía tradicional, también será limitado cuando el microscopio se coloca entre el cirujano y el campo quirúrgico; sin embargo, el microscopio crea una vista mucho mejor del campo quirúrgico mediante un aumento apropiado y una iluminación altamente enfocada. ${ }^{18}$

\section{VENTAJAS DEL USO DEL MICROSCOPIO}

El microscopio no sólo aporta mejoras en los tratamientos, también se ha demostrado su relevancia para la visión y ergonomía del profesional. El posicionamiento adecuado para el operador, el paciente y el asistente es absolutamente necesario. ${ }^{19}$ La posición correcta del operador para casi todos los procedimientos de endodoncia es directamente detrás del paciente, en la posición de las 11 o las 12 en punto, aun cuando pueden parecer más cómodas otras posiciones cuando se aprende a usar un MOD. ${ }^{19} \mathrm{La}$ observación del campo a través de los oculares elimina la visión colateral. La iluminación coaxial proporciona una iluminación más homogénea, lo que permite visualizar el campo operatorio sin presencia de sombras; por lo tanto, se elimina información no relevante que mejora la visión y la concentración minimizando la fatiga ocular. ${ }^{2}$

\section{DESVENTAJAS ASOCIADAS AL MICROSCOPIO OPERATORIO}

Las dos principales desventajas que se mencionan en la literatura se enfocan al costo del instrumento y la dificultad que puede generar aprender a usarlo, generando una necesaria curva de aprendizaje. El periodo de ajuste es más pronunciado para dispositivos con mayor aumento. La coordinación mano-ojo se debe volver a aprender a medida que la endodoncia se realiza con visión indirecta.

El microscopio es significativamente más caro que otros instrumentos de magnificación. Sin embargo, teniendo en cuenta los beneficios que aporta, el tema costobeneficio apoya el hecho de adquirir esta tecnología. Una última posible desventaja es que se debe diseñar un espacio organizativo en el operatorio dental que permita girar en torno a un principio ergonómico que no es tan fácil de conseguir en espacios operatorios que fueron diseñados sin tener en cuenta la presencia del MOD. ${ }^{5}$

Puede parecer obvio que el uso de magnificación sólo podría ser ventajoso, aumentando así el éxito a largo plazo del tratamiento endodóncico; no obstante, aún es difícil encontrar estudios relevantes que puedan demostrar que esto es necesariamente cierto. Se desconoce si la magnificación afecta el pronóstico del tratamiento y cómo lo hace, considerando la gran cantidad de factores que pueden tener un impacto significativo en el éxito de la terapia endodóncica. ${ }^{20}$

\section{CONCLUSIÓN}

El odontólogo debe analizar qué tan conveniente es aprender a usar un microscopio en la práctica, las ventajas y desventajas de su adquisición, así como las dificultades que pueden surgir al aprender a usarlo, además del costo que genera adquirir este recurso. La calidad de los tratamientos no depende sólo del microscopio, se puede usar éste en todos los tratamientos realizados, pero si el operador no tiene los conocimientos básicos, el microscopio sólo será una manera de ver mejor.

La revisión de literatura confirma que las aplicaciones del microscopio aportan mejoras en el procedimiento endodóncico, destacando que se pueden realizar tratamientos en situaciones clínicas que anteriormente serían descartados. 
El uso del microscopio ha generado la creación de nuevos protocolos, de diseños necesarios para poder maniobrar con él en un consultorio de manera ergonómica, e incluso una discusión sobre si adquirirlo y aprender a usarlo vale la pena.

El consenso generalizado entre los profesionales en la actualidad habla de un aumento en la competitividad usando el instrumento, más allá del simple marketing y se cree que en un futuro su uso será, si no obligatorio, sí un estándar en una práctica endodóncica que se precie de ser completa y actual.

\section{REFERENCIAS}

1. Sánchez Lera RM, Oliva García NR. Historia del microscopio y su repercusión en la Microbiología. Rev Hum Med. 2015; 15 (2): 355-372.

2. Moradas Estrada M. Importancia de la magnificación en odontología conservadora: revisión bibliográfica. Av Odontoestomatol. 2017; 33 (6): 283-293.

3. Malfaz-Vázquez JM. Aplicaciones del microscopio en la Endodoncia actual. RCOE. 2002; 7 (3): 301-310.

4. Campo Barrau N. Microscopia en endodoncia. Barcelona, España: 2010. Disponible en: https://microendodoncia.files.wordpress. com/2013/03/microscopia_en_endodoncia.pdf

5. Carr GB, Murgel AF. The use of the operating microscope in endodontics. Dent Clin N Am. 2010; 54 (2): 191-214.

6. Nemeh AA, Aqrabawi J. Magnification in endodontics a literature review. J Dent Biomater. 2014; 9 (2): 14-21.

7. Gondim Jr E, Setzer F. The dental operating microscope in endodontics. Int J Microdent. 2010; 2 (1): 20-27.

8. Sánchez G, Alegría M, Pesce D, Alcántara R. Localización de conductos radiculares: Visión directa $\mathrm{v} / \mathrm{s}$ microscopio quirúrgico. Estudio in vitro. J Oral Res. 2012; 1 (1): 10-14.

9. Ageste Duque TM, Herrera DR, Ferraz CC, Zaia AA, Almeida JF, Gomes BP. Localización efectiva de un segundo conducto radicular en incisivos inferiores mediante magnificación, radiografía y diafanización. Rev Estomatol Heredia. 2013; 23 (2): 57-62.

10. Monteiro-Bramante C, Torres M, Silva Bramante A, Gomes de Moraes I, Bernardineli N, Brandão Garcia R et al. Determinación de la frecuencia del conducto mesiopalatino en la raíz mesiovestibular de molares superiores. Análisis comparativo con dos métodos de evaluación. Med Oral. 2004-2005; VI y VII (especial): 87-90.

11. Ranganath JG. Endodontic management of a two rooted, three canaled mandibular canine with a fractured instrument. J Conserv Dent. 2014; 17 (2): 192-195.

12. Gencoglu N, Helvacioglu D. Comparison of the different techniques to remove fractured endodontic instruments from root canal system. Eur Dent J. 2009; 3 (2): 90-95.

13. Espinosa-Torres A. Sellado de perforaciones por desgaste en la furca, reporte de dos casos con control a cinco años. Rev Nal Odontol. 2011; 3 (6): 20-24.

14. Espinosa Torres A. Microcirugía periapical. Reporte de un caso. Rev ADM. 2011; 68 (2): 89-92.

15. Giménez del Arco ML, Lloveras V, García Puente C. Microcirugía endodóntica ante una lesión periapical persistente. Odontoespacio. net. 2016; 6 (3): 11-19.

16. Espinosa Torres A. Accidente operatorio en furca resuelto con microcirugía. Presentación de un caso con control a 30 meses. Canal Abierto. 2016; 34: 36-39.

17. Taschieri S, Weinstein T, Tsesis I, Bortolin M, Del Fabbro M. Magnifying loupes versus surgical microscope in endodontic surgery: A four-year retrospective study. Aust Endod J. 2013; 39: 78-80.

18. Abbott PV. Endodontics-current and future. J Conserv Dent. 2012; 15: 202-205.

19. Low JF, Mohd Dom TN, Baharin SA. Magnification in endodontics: A review of its application and acceptance among dental practitioners. Eur J Dent. 2018; 12: 610-616.

20. Del Fabbro M, Taschieri S, Lodi G, Banfi G, Weinstein RL. No evidence that magnification devices improve the success of endodontic therapy. Evid Based Dent. 2016; 17: 84-85.

Conflicto de intereses: Los autores declaran no tener ningún conflicto de intereses.

Aspectos éticos: Ninguno.

Financiamiento: Ninguno.

Correspondencia:

Dr. Ricardo Oliva-Rodríguez

E-mail: ricardo.oliva@uaslp.mx 